\title{
Simulating
}

\section{Large-Area Plasma Displays}

\author{
J.-P. Boeuf ${ }^{1}$, H. Doyeaux ${ }^{2}$ \\ ${ }^{1}$ Centre de Physique des Plasmas et Applications de Toulouse, \\ CNRS-Université Paul Sabatier, Toulouse, France \\ ${ }^{2}$ Thomson Tubes Electroniques, Moirans, France
}

\section{Sophisticated numerical models to provide an improved understanding of physical phenom- ena play a major role in advancing the key technology of plasma display panels.}

"Plasma fever is spreading". This quote from S. Mikoshiba in a recent issue of Information Display [10 (1994) 21] suggests that more and more people feel that plasma display panels (PDP) are now the most promising technology for large size (more than 40-inch diagonal) television monitors. This renewed enthusiasm for PDP technology is due partly to the significant progress that has been made with colour plasma displays over the last five years, and partly to the persistent difficulties in manufacturing large-size, zerodefect, thin-film transistor matrixes for liquid-crystal displays, the main technology used for compact and lightweight units. Plasma display panels are now prominent competitors in the market for large-size displays for televisions, as illustrated by the recent decision of several Japanese companies to build manufacturing plants for 40-inch PDPs.

\section{Large-Screen Technologies}

The cathode ray tube (CRT) today represents by far the dominant display technology in consumer TV applications. CRTs provide excellent performance at low cost, but their footprint and weight

J.-P. Boeuf has been a Directeur de Recherche at the CNRS-Université Paul Sabatier Centre de Physique des Plasmas et Applications de Toulouse since 1993. He graduated from the Ecole Supérieure d'Electricité (Paris) in 1977 and obtained his Doctorat d'Etat in plasma physics from the Université de Paris XI, Orsay, in 1985. He worked as a Visiting Assistant Professor at Texas Tech University in the USA before joining the CNRS Laboratoire de Physique des Décharges at the Ecole Supérieure d'Electricité in 1983.

H. Doyeux joined the Plasma Displays Group of Thomson Tubes Electroniques, ZI Centr'Alp, F-38430 Moirans, in 1990, where he is in charge of developing colour plasma display panel technology, having been responsible for the development of high-voltage power supplies and colour cathode ray tubes. He was educated in Paris at the Ecole Polytechnique and at the Ecole Supérieure d'Electricité. become prohibitive for screens with a diagonal size larger than about 35 inches.

Liquid crystal displays (LCD) have made possible the widespread use of portable computers, where limits on weight and power consumption are strict. Technical progress and huge investments in this technology are leading to the commercialization of portable TV sets at a reasonable price, but owing to manufacturing considerations, LCDs seem to be limited for many more years to low- and medium-size display applications.

Other flat display technologies such as electroluminescent and field-emission displays may compete in the future with LCDs at small screen sizes, but they have not yet proved feasible for large sizes.

Plasma display panels provide at the moment the basis for the most attractive large, wall-hanging TV displays. They are characterized by wide viewing angles, a large range of colours, high contrast ratio, scanning speeds which are rapid enough for TV, and manufacturing capabilities for large-area substrates. The depth and and $10 \mathrm{~kg}$ for a PDP, as compared to 100 $\mathrm{cm}$ and $150 \mathrm{~kg}$ for a CRT of the same size (power consumption on the order of 350 W for both). Fig. 1 illustrates this favourable comparison for a 22 -inch PDP and a weight of a 40-inch display would be $5 \mathrm{~cm}$

CRT television of about the same size.

For very large sizes (more than 50- or 6o-inch), projection displays - either CRT projection or LCD projection - offer the only immediate, practical solution for TV applications.

\section{Principles}

In plasma display panels, the light of each picture element is emitted from a plasma created by an electric discharge (see insert). The dimensions of the discharge are around $100 \mathrm{~mm}$ at a pressure of a few hundred Torr, and the voltage applied between the electrodes is in the 100-200 V range. In the simplest configuration, a plasma display panel consists of two glass plates, each with parallel arrays of thin conducting strips deposited on their surfaces; these strips serve as the electrodes. The plates are sealed, together with their electrode arrays at right angles to each other, and the gap between the plates is filled with a rare-gas mixture. Each pixel at the intersection of a line and a column electrode can be illuminated independently when a voltage pulse is applied between the two electrodes. The voltage pulse leads to the breakdown (see insert) of the gas and to the formation of a weakly ionized plasma which emits visible or ultra-violet light.

The visible light from the discharge is used in monochrome displays. In colour displays, UV emission from the discharge is used to excite phosphors in the three fundamental colours (one pixel of the screen includes three discharge cells). Typical rare-gas mixtures used in colour PDPs are neon-xenon and helium-xenon, where UV photons are emitted at $147 \mathrm{~nm}$ by xenon excited atoms $\mathrm{Xe}^{*}\left({ }^{3} \mathrm{P}_{1}\right)$ at $150 \mathrm{~nm}$ and $173 \mathrm{~nm}$ by xenon excimer molecules $\mathrm{Xe}_{2}^{*}\left(\mathrm{O}_{\mathrm{u}}^{+}\right), \mathrm{Xe}_{2}^{*}\left({ }^{3} \Sigma \mathrm{u}^{+}\right)$and $\mathrm{Xe}_{2}^{*}\left({ }^{1} \Sigma \mathrm{u}^{+}\right)$. The choice of the percentage of the buffer gas (helium or NEON) results from a compromise between a lower operating voltage (more buffer gas) and a larger UV produc-

Fig. 1. A photograph comparing a 23-inch cathode ray tube display, on the left, with a 22 -inch plasma display panel, on the right.

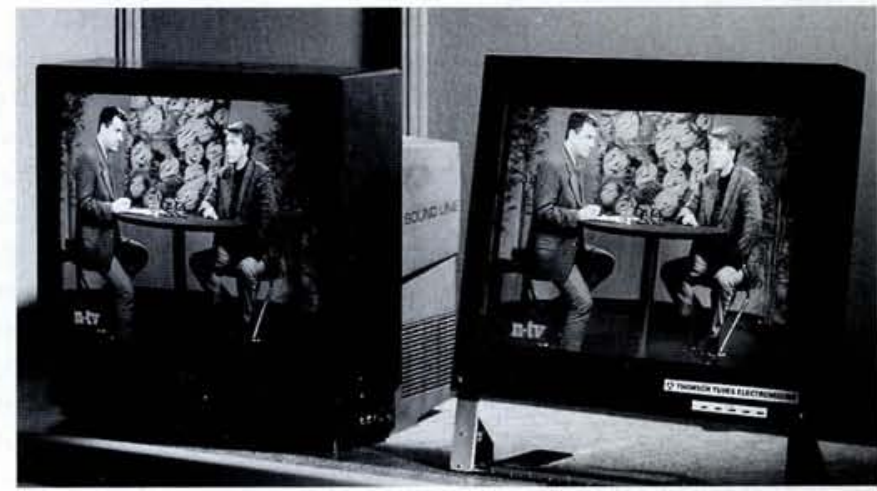


tion (more xenon). The excited species are created in the discharge by electron impact excitation of xenon atoms in the ground state followed by excitation transfer reactions. The large sheath electric field imparts to the electrons the energy needed to excite atomic xenon.

PDPs are available with either alternating current $(\mathrm{ac})$ or direct current $(\mathrm{dc})$ driving circuitry. In dc PDPs, the electrodes are in contact with the gas mixture while in ac devices the electrodes are covered with a dielectric layer. We focus below on ac PDPs.

The dielectric layers are coated with a magnesium oxide $(\mathrm{MgO})$ layer. The role of the $\mathrm{MgO}$ film is to protect the dielectric layers and to decrease the discharge voltage owing to the large secondary electron emission coefficient of $\mathrm{MgO}$ under bombardment by neon ions. In some PDPs, the cell geometry includes dielectric barrier ribs parallel to the electrodes on one plate in order to physically separate adjacent cells and to avoid cross-talk (see Fig. 2).

In operation, a square ac waveform (sustaining voltage) is applied continuously across the line and column electrodes. The amplitude of this voltage is less than the breakdown voltage. To turn an element on, an additional voltage is superimposed on the sustaining waveform, between the line and the column defining the cell: a discharge forms and UV photons are emitted. The creation of this discharge results in the build-up of charge (memory charge) on the dielectric layers which then sets up a voltage opposite to the applied voltage. This reduction of the gap voltage leads to the extinction of the discharge. On reversing the polarity, the voltage due to the memory charge adds to the applied voltage and the discharge cell fires again. A pulsed discharge is therefore initiated in the cell at each half cycle when the pixel is on. To erase the pixel, another voltage pulse is superimposed on the sustaining voltage, of an amplitude such that the memory charge is cancelled after the discharge pulse. Typical sustaining frequencies are on the order of $50 \mathrm{kHz}$, and a typical pulse duration for a $90 \% \mathrm{Ne}-10 \%$ Xe mixture with a $100 \mu \mathrm{m}$ gap length at 600 Torr is $20 \mathrm{~ns}$. The "on" state is therefore a succession of 20 ns discharge pulses at $100 \mathrm{kHz}$. The pulse duration can be strongly affected by the gas mixture and geometry.

The cells can only be in "on" or "off" states, and it is impossible to modulate the luminous intensity of a discharge pulse. Grey scale (variable intensity) can be

\section{Discharge Below Breakdown is Fundamental}

Gas breakdown is the transition from the insulating state to a conducting state of a gas. When a voltage is applied in a gas between two electrodes, the free electrons present in the gap are accelerated and can reach energies high enough to excite and ionize the gas molecules (excitation and ionization thresholds are in the $10-20 \mathrm{eV}$ range). This leads to the formation of electron avalanches. The positive ions resulting from the ionization events are accelerated to the cathode and can extract secondary electrons from the cathode surface. Gas breakdown occurs when the system is self-sustained, i.e., when each electron reaching the anode is, on average, replaced by one electron emitted by the cathode under ion (or photon) impact.

This description of gas breakdown is valid only for $p d$-products (pressure $p$ times gap length $d$ ) less than a few 10 Torr.cm, conditions which are typical of plasma displays. The breakdown voltage is a function of the $p d$-product, of the gas mixture (the electron multiplication in the gap depends on the electron-atom cross section) and of the cathode material (which defines the value of the secondary electron emission under ion impact).

If the current is limited with an external resistor, the discharge can operate in a low current, non-emissive regime (the so-called Townsend regime) where space-charge distortion of the applied electric field is negligible. In this regime, the positive ion density in the gap is much larger than the electron density (electrons are much more mobile than ions). When the current is progressively increased by decreasing the value of the external resistor, the accumulation of positive ions in the gap induces a field distortion. This field distortion changes the electron energy balance and generally tends to increase the electron energy and multiplication, which leads to a further increase in the discharge current and ion density, and a decrease in the gap voltage.

When the positive ion space-charge field is on the same order as the geometric field, electrons can no longer diffuse freely in the gap and a quasi-neutral

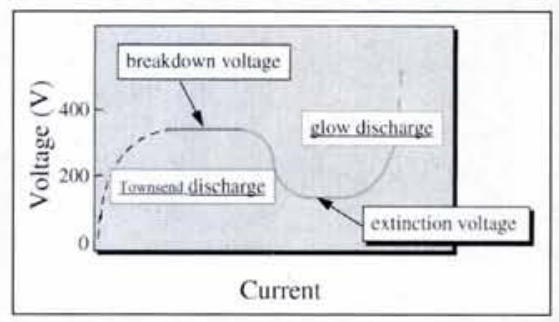

Current-voltage characteristics of a dc glow discharge. The fact that the discharge can operate at a lower voltage than the one required to initiate breakdown of the gas is fundamental, and lies at the origin of the bistable operating voltage margin of PDPs..

region forms on the anode side of the discharge, where electrons and ions diffuse at the same speed (ambipolar diffusion). The electric field in the quasineutral region (the "plasma") decreases while the electric potential is redistributed in the region between the cathode and the plasma ("the sheath").

The system evolves with the plasma region expanding and the sheath contracting until the self-sustaining condition is once again fulfiled. This new regime corresponds to the glow discharge and is characterized by intense light emission due to electron impact excitation of the gas atoms or molecules. The glow discharge can operate at voltages below the breakdown voltage, as shown in the figure, the minimum voltage depending on the gas mixture and cathode material (typically $200 \mathrm{~V}$ ). The minimum voltage is generally called the extinction voltage in direct current PDPs.

The fact that the discharge can operate at a lower voltage than the one required to initiate breakdown of the gas is fundamental and is at the origin of the bistable operating voltage margin of PDPs. The existence of the margin and threshold properties of the discharge make possible the addressing of specific cells in an array without changing the state of cells not specifically addressed. The concept of margin is also valid for alternating current PDPs, where the discharge formation is as described above. However, the discharge is quickly quenched in alternating current PDPs owing to the charging of the dielectric layers above the electrodes. 

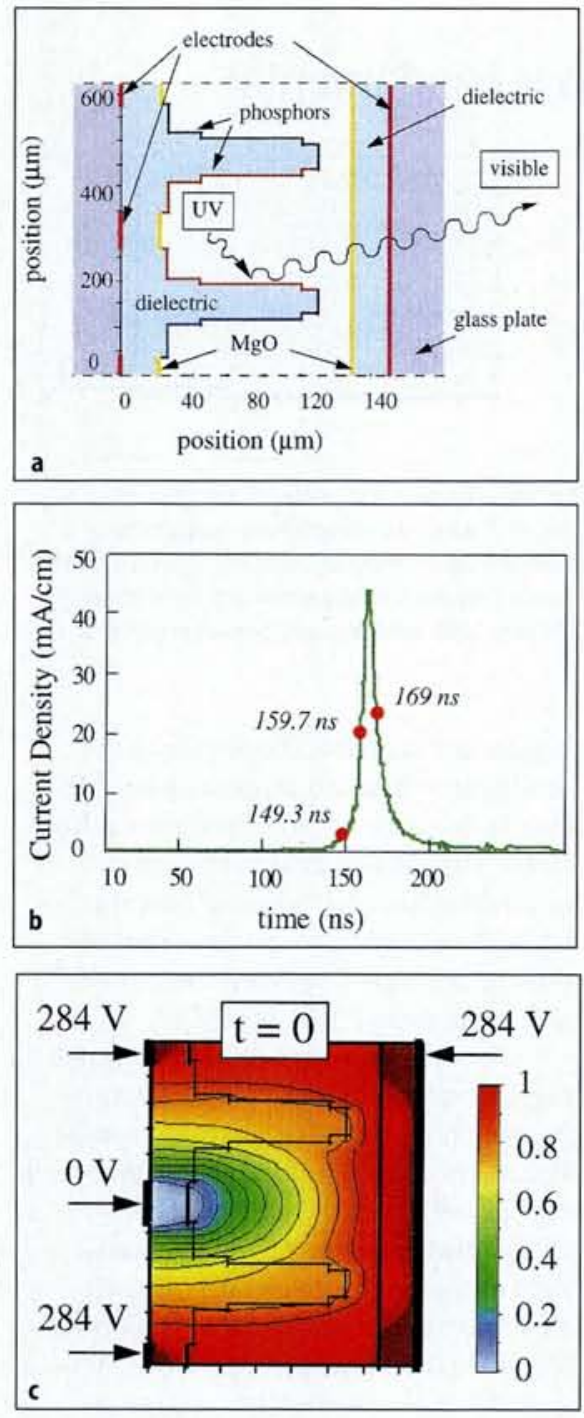

achieved by using a duty cycle modulation, i.e., by modulating the duration of the "on" state (the apparent brightness is the temporal average of the brightness corresponding to one discharge pulse).

\section{Particle and Photon Transport}

The physical mechanisms occurring in a PDP cell can be described using numerical models. These models are based on equations describing electron and ion transport in a weakly ionized gas, coupled with Poisson's equation for the electric field and with boundary conditions (charging of the dielectrics; applied voltage). Under PDP conditions, a macroscopic approach to charged particle transport where continuity, momentum and energy transport equations are used is accurate enough. The electron transport coefficients (mobility; diffusion) and the excitation and ionization frequencies can be tabulated as a function of electron energy by solving the Boltzmann equation

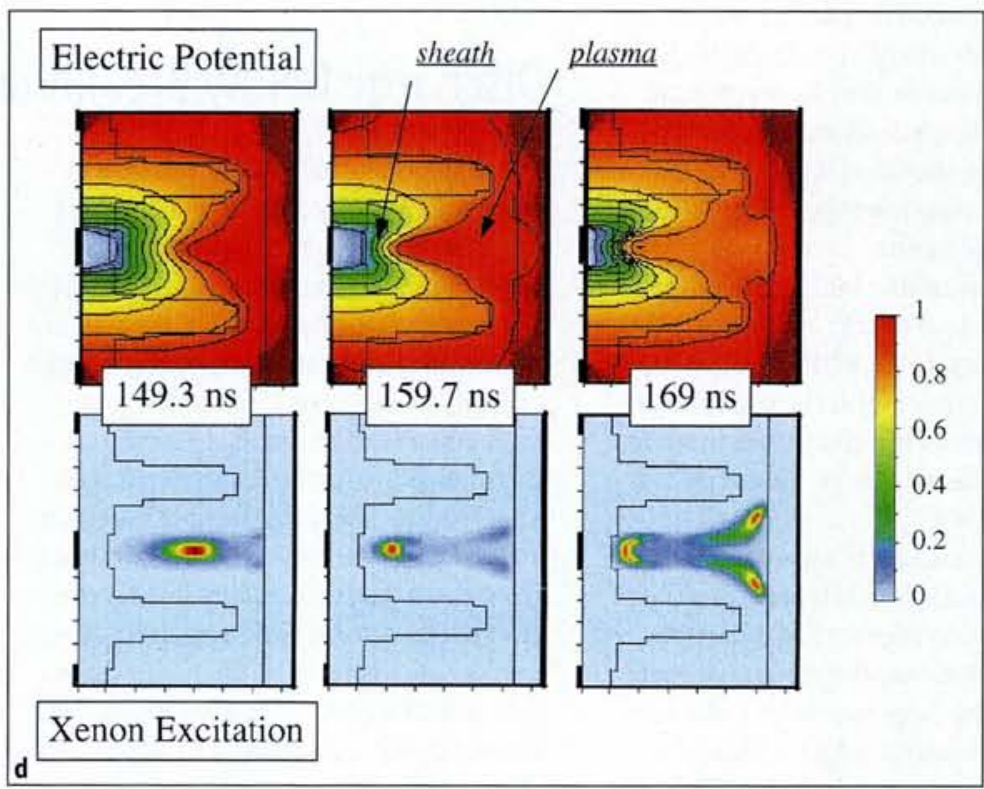

Fig. 2. An example of a numerical study of discharge formation in a PDP cell. The calculated discharge current as a function of time is plotted in (b) for the PDP cell illustrated schematically in (a) showing the dielectric barrier ribs that physically separate adjacent cells. An entire cell and one-half of the two adjacent cells are represented; the gas mixture is $10 \%$ xenon - $90 \%$ neon and the pressure is 560 Torr. The initial voltage of the right-hand electrode is set to $284 \mathrm{~V}$; that of the left-hand centre electrode to $0 \mathrm{~V}$ and the two outer left-hand electrodes to $284 \mathrm{~V}$. The spatial distribution at time $t=0$ of the electric potential (contour lines to guide the eye; colours blue through to red corresponding to the electric potential increasing from $0 \mathrm{~V}$ at the centre of the left-hand electrode to the full $284 \mathrm{~V}$ of the right-hand electrode) are shown in (c). The calculated electric potential and xenon excitation during the evolution of the discharge are plotted in (d) for three different times corresponding to the three points indicated on the discharge current curve. Fig. 2(b). Note that the xenon excitation is also represented as blue through to red for increasing excitation.

under homogeneous conditions, and used in the macroscopic transport equations.

The solution of electron and ion transport coupled with Poisson's equation provides the time variation of current and voltage across the discharge as well as the variation with space and time of the charged particle densities, electric field, excitation, and ionization rates. Knowing the space and time variations of the excitation rate of the various electronic states of xenon, it is possible to build a kinetic model to study the evolution of the population of these states and the resulting photon production and transport. The rates for the reactions involved in such a model are reasonably well known because of the numerous studies on gas discharge lasers (e.g., excimer lasers) involving raregas mixtures. The photon transport model is slightly complicated owing to imprisonment of resonant radiation (the photons emitted in the de-excitation of atomic excited xenon can be absorbed by xenon atoms in the ground state and re-emitted several times before exiting the cell).

Fig. $2 \mathrm{~d}$ illustrates the development of a plasma discharge in a typical PDP cell. The calculated electric potential and the xenon excitation during the evolution of the dis- charge are plotted for three different times corresponding to the three points on the discharge current curve. The discharge is initiated between the centre-left electrode and the right-hand electrode, and after $149.3 \mathrm{~ns}$ the plasma has already formed on the anode side of the cell (right-hand electrode). The location of the plasma corresponds to the region where the potential contours are well separated (the plasma is a conducting medium and the plasma field is low). The applied potential then redistributes in the sheath region on the cathode side of the cell. The maximum amount of xenon excitation occurs at the plasma sheath boundary where the electrons are relatively energetic. As time goes on, the plasma expands while the sheath contracts, and the maximum moves toward the cathode side of the cell. While the current flows through the cell the dielectric layers charge up and the voltage in the gap decreases (see the redistribution of the potential in the dielectric at time $t=169$ $n s)$. Note also the distribution of xenon excitation at $t=169$ ns showing a spreading of the plasma along the right-hand dielectric layer, owing to the charging of the dielectric surface. The negative charge accumulated on the surface around the 
discharge axis repels incoming electrons, which are driven away from the axis by the resulting electric field. Xenon excitation is, however, smaller at $t=169 \mathrm{~ns}$ than at $t=$ 159.7 ns (i.e., the centres of the excited regions appear in a less intense red colour owing to the significant decrease in the discharge current).

\section{Research Issues}

A weakly ionized plasma represents a complex system in which electrons, positive ions, excited species, and photons interact together and with the electric field. A better qualitative and quantitative understanding of the physical phenomena occurring in a PDP cell is being achieved by developing sophisticated, user-friendly numerical models.

The luminous efficiency of PDPs has been considerably increased in the last few years, but it is still relatively low (on the order of $1 \mathrm{~lm} / \mathrm{W}$ ) and one of the issues in PDP technology is the improvement of this parameter. The low efficiency is partly due to the fact that a large proportion of the electrical energy is dissipated by positive ions in the high-field sheath region, through collisions with neutral atoms or with the walls. Improvement of the luminous efficiency can be achieved by improving the cell's geometrical design, optimizing the gas mixture and using phosphors with a higher quantum efficiency. Lowering the operating voltage is also an important issue, and could be achieved by increasing the secondary electron emission of the film covering the dielectric layers and the gas mixture. This is mainly a cost issue since the technology of high-voltage $(200 \mathrm{~V})$ integrated driver circuits is now under control.

Addressing speed is another important issue because it impacts on the greyscale rendition, and more generally on image quality (moving picture rendition). Improvements in this area require action both on the physics of the PDP (time response of the cells) and on the electronic driving circuit.

These research issues invariably require a better understanding of the physical mechanisms involved in plasma display panels, so sophisticated but userfriendly numerical modelling of the discharge cells represents a way to achieve substantial technical improvements.

The Panilevé Property, A Century Later School - Cargèse, France; 3-22 June 1996

Contact: R. Conte, DRECAM-SPEC, CE Saclay, F-91191 Gif-sur-Yvette - E-mail: conte@amoco.saclay.cea.fr Tel.: +33-1- 69087349 - Fax: +33-1-69088786

\section{Painlevé a Century Later}

The signal which propagates along a typical optical fibre used for trans-oceanic communication comprises a solitary wave, with an exact analytic expression depending on very few adjustable parameters. Tremendous effort is needed to amplify the signal every $400 \mathrm{~km}$. Present theory predicts the probable existence of an exact, essentially attenuation-free solution with more free parameters. Its discovery, a difficult task, will necessarily involve methods for integrating nonlinear differential equations that were articulated a century ago by the French mathematician Paul Painlevé, whose contributions will be marked by a Cargèse school this spring. In covering both the old, but not obsolete, methods as well as modern extensions to physics, it will be honoured by the presence of Professor Bureau, the last disciple of the Painlevé school, and by Professor Kruskal, the co-discoverer of the soliton.

The Painlevé property states that in order to define a function, a differential equation must have a general solution without any branching around the singular points whose positions depend on the initial conditions. All linear equations have this property and provide the elements for building analytic expressions (polynomials, exponentials, trigonometric, Bessel, etc., functions). Nonlinear equations, on the other hand, provide very few, new functions. For instance, first order only defines one new function, the elliptic function (the famous inverse function $u(x)$ of the so-called elliptic integral $x(u)$ ruling the motion of the pendulum).

It was the systematic examination of all differential equations of second order which in 1898-1906 led Painlevé and his student Gambier to the discovery of six new functions defined by a differential equation, the first - $\mathrm{P}_{1}$ - being simply $d^{2} u / d x^{2}=6 u^{2}+x$. Painlevés feat for long only excited the curiosity of a few mathematicians, since the fashion turned to more abstract mathematics.

The renewal of interest dates backs to the end of the 1960 s, pushed by three apparently distinct domains of physics. First, the discovery of the soliton in 1965 initiated the booming domain of integrable evolution equations, i.e., those admitting elastic collisions of quasiparticles called multi-solitons [1]. The link with Painlevé is that, whenever such an integrable equation admits what is technically called a noncharacteristic reduction to an ordinary differential equation (ODE), this ODE has the Painlevé property. One example is the Korteweg-de Vries equation for $U(X, T)$,

$$
U_{T}+U_{X X X}-12 U U_{X}=0,
$$

and its reduction to ( $\left.\mathrm{P}_{1}\right)$ for $u(x)$,

$$
u(x)=U(X, T)+T ; x=X-6 T^{2} .
$$

Second, statistical physics and field theory have provided a number of exactly solvable models, in which typically the free energy or some correlation function, as a function of say the temperature, obeys a Painlevé equation. A celebrated example is the two-dimensional Ising model while the self-dual Yang-Mills equations, fundamental to field theory, admit a reduction to the master Painlevé equation P6.

Third, the advent of computers has popularized the observation that dynamic systems such as the Lorenz model of atmospheric circulation,

$$
x^{\prime}=\sigma(y-x) ; y^{\prime}=-x z+r x-y ; z^{\prime}=x y-b z
$$

although deterministic, i.e., first order in time, generically exhibit chaotic behaviour. However, for some values of the control parameters $(b, \sigma, r)$, easily found by the so-called Painlevé test, they may have the Painlevé property. For instance, the case of $b=0, \sigma=1 / 3$ is integrable with $\mathrm{P}_{3}$.

The renewal in interest has produced feedback in two major directions: the extension of the theory of Painlevé to partial differential equations [2] and, more recently, to finite difference equations [3] under the impulse of statistical lattice physics. In our youth we learnt many recipes for integrating nonlinear differential equations. But simple and powerful methods to achieve the goal when integration is possible were developed a century ago in Painlevé's famous Leçons de Stockholm [4]. This text, although temporarily - one hopes - out of print, remains a reference.

R. Conte, CE Saclay

References,

[1] Ablowitz M.J., Clarkson P.A., Solitons, nonlinear evolution equations and inverse scattering, LMS Lecture Note Series (Cambridge University Press, 1991). [2] Weiss J., Tabor M. \& Carnevale G., J. Math. Phys. 24 (1983) 522 .

[3] Brézin E., Kazakov V.A., Phys. Lett. B 236 (1990) 144; Gross D.J., Migdal A.A., Phys. Rev. Lett. 64 (1990) 127.

[4] Oeuvres de Paul Painlevé, 3 vols. (Editions du CNRS, Paris; 1973, 1974, 1976). 